\title{
Enhanced publications: um estudo da plataforma Public Library of Science (PLOS)
}

\author{
Enhanced publications: a Public Library of Science (PLOS) platform study \\ Edilaine Avila ${ }^{1}$, Cláudia Regina Ziliotto Bomfá \\ ${ }^{1}$ Universidade Federal de Santa Maria (UFSM), Santa Maria, RS, Brasil. ORCID: https://orcid.org/0000-0002-2561-1858 \\ 2 Universidade Federal de Santa Maria (UFSM), Santa Maria, RS, Brasil. ORCID: https://orcid.org/0000-0001-9398-3146
}

Autor para correspondência/Mail to: Edilaine Avila, edilaineavila@gmail.com

Recebido/Submitted: 28 de setembro de 2020; Aceito/Approved: 14 de dezembro de 2020

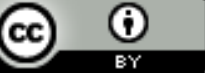

Copyright (c) 2021 Avila \& Bomfá. Todo o conteúdo da Revista (incluindo-se instruções, política editorial e modelos) está sob uma licença Creative Commons Atribuição 4.0 Internacional. Ao serem publicados por esta Revista, os artigos são de livre uso em ambientes educacionais, de pesquisa e não comerciais, com atribuição de autoria obrigatória. Mais informações em http://revistas.ufpr.br/atoz/about/submissions\#copyrightNotice.

\begin{abstract}
Resumo
Introdução: Identifica e apresenta quais recursos das enhanced publications podem ser utilizados pelos periódicos científicos digitais. Apresenta, como objeto de análise, a Public Library Of Science (PLOS), a partir da seguinte questão norteadora: quais características identificam uma publicação científica como uma enhanced publication e como essas particularidades são utilizadas pelos periódicos científicos? Método: Pesquisa de natureza qualitativa, em nível exploratório que, com um levantamento, culminou em uma análise de conteúdo dos dados encontrados. Resultados: Identifica e analisa as seguintes características que definem uma publicação como enhanced publication: hipertextualidade, multimidialidade, interatividade, memória, personalização e ubiquidade. Conclusão: As características de uma enhanced publication foram definidas, além de ter sido demonstrado como a PLOS trabalha esses recursos com o intuito de ampliar sua visibilidade.
\end{abstract}

Palavras-chave: Comunicação; e-Science; Produção Editorial; Enhanced Publications; Visibilidade.

\begin{abstract}
Introduction: It identifies and presents which enhanced publications resources can be used by digital scientific journals. It presents the Public Library Of Science (PLOS) as an object of analysis, based on the following guiding question: which characteristics identify a scientific publication as an enhanced publication and how are these particularities used by scientific journals? Method: Qualitative research, at the exploratory level, which, with a survey, culminated in a content analysis of the data found. Results: It identifies and analyses the following features that define a enhanced publication: hypertextuality, multimedia, interactivity, memory, personalization and ubiquity. Conclusion: The characteristics of an enhanced publication have been defined, and it has been demonstrated how PLOS works with these resources to increase its visibility..
\end{abstract}

Keywords: Communication; e-Science; Editorial Production; Enhanced Publications; Visibility.

\section{INTRODUÇÃO}

Desde a sua aparição, ainda no século XVII, os periódicos científicos têm papel central no contexto da Comunicação Científica, pois são considerados "o principal canal formal de disseminação da Ciência" (Valerio, 2005 como citado em Ambinder (2012, p. 40)). Entretanto, com a evolução das tecnologias, principalmente com o surgimento da internet, a comunidade científica vem observando a necessidade de novos modelos para as publicações, que apresentem maior interatividade e que acompanhem as mudanças que o ambiente digital trouxe para as relações comunicacionais.

Nesse sentido, as Tecnologias da Informação e Comunicação (TICs) e os movimentos de abertura da ciência (arquivo aberto, acesso aberto, dados abertos e ciência aberta) têm imposto mudanças na forma de disseminar os resultados de pesquisa, requerendo recursos informatizados. Valerio (2006) enumera esses recursos como: disponibilização de links, novos canais de comunicação, motores de busca, softwares, mapas de dados, entre outros. Como forma de atender às necessidades da comunidade científica ante às mudanças mencionadas anteriormente, surgem as chamadas enhanced publication, definidas por Gomes (2014, p. 200) como "uma publicação enriquecida a partir de três categorias de informações: os dados da pesquisa; os materiais extras; e os dados de pós-publicação". Enhanced publications despontam como uma evolução dos estudos sobre repositórios feitos por Woutersen-Windhouwer, Brandsma, Hogenaar, e Hoogerwerf (2009), com progresso nos conceitos posteriormente.

Assim, enhanced publication, também conhecida como publicação ampliada, pode representar "uma coleção de objetos e diversas relações mútuas, ultrapassando, pois, a característica 'unilateral' condizente com as publicações ditas 'tradicionais'" (Gomes, 2014, p. 200). Uma de suas principais características, portanto, é a sua capacidade de facilitar o acesso às fontes de pesquisa, promovendo o cruzamento de informações até então isoladas em seus universos de produção. Deste modo, a plataforma Public Library Of Science (PLOS) pode ser considerada como uma enhanced publication, na medida em que oferta recursos das publicações ampliadas. Tanto que Woutersen-Windhouwer e Brandsma (2009) revelam que a PLOS atende as três principais características das 
enhanced publications, como dados de pesquisa, materiais extras e comentários pós-publicação. A PLOS foi fundada em 2001 como uma organização sem fins lucrativos de acesso aberto, com a missão de acelerar o progresso em Ciência e Medicina, levando a uma transformação na comunicação da pesquisa científica. ${ }^{1}$

Assim, o presente artigo apresenta os resultados de uma pesquisa realizada na PLOS, cujo objetivo é identificar e apresentar quais recursos das enhanced publications são utilizados por esta plataforma. Além disso, tem-se também o objetivo de contribuir com a discussão sobre esse tipo de publicação, ofertando insumo a avaliações de outras iniciativas e descrevendo os recursos disponíveis pela PLOS.

\section{MARCO TEÓRICO}

Uma das principais características da comunicação em rede é a produção de muitos para muitos, que é entendida a partir de múltiplos atores produzindo inúmeras mensagens para um grupo vasto de receptores. Nesse contexto, não mais se estabelece o fluxo clássico de comunicação, em que a mensagem era produzida de um para um, ou de um para muitos. Atualmente, tem-se uma gama de transmissões de conteúdos acontecendo ao mesmo tempo, com rápida codificação e decodificação.

Com isso, cresce exponencialmente a cultura da colaboração em rede, na qual a produção de conteúdo é estimulada entre comunidades virtuais ou redes de relacionamento, para as quais os membros se reúnem em torno de afinidades comuns com o intuito de compartilhar informações. Esses ambientes colaborativos foram ampliados a partir da web 2.0, que se diferencia por sites ou aplicativos com baixo custo de desenvolvimento. Neste caso, o conteúdo surge de baixo para cima a partir do relacionamento entre os participantes, além de combinar conteúdo de mais de uma plataforma para produzir experiência integrada (Spyer, 2007).

Neste contexto, para Bardi e Manghi (2014, p. 1), as enhanced publications são "publicações digitais que consistem em uma parte narrativa obrigatória (a descrição da pesquisa realizada) mais partes 'relacionadas', como conjuntos de dados, outras publicações, imagens, tabelas, fluxos de trabalho, dispositivos". As enhanced publications têm, portanto, a finalidade de tornar possível o processamento dos dados, de forma a garantir que as informações sejam facilmente pesquisadas no ambiente web (Degkwitz, 2016).

Sales, Sayão, e Souza (2013) afirmam que, em 2007, o Digital Repository Infrastructure Vision for European Research - II (DRIVER-II), repositório digital europeu, publicou um relatório sobre objetos digitais, no qual definiu o termo enhanced publication ou publicação ampliada como: "objetos digitais compostos que combinam e-prints ${ }^{2}$ com um ou mais recursos de dados, um ou mais registros de metadados, ou qualquer combinação destes" (Sales et al., 2013, p. 5).

Woutersen-Windhouwer e Brandsma (2009) propõem que o conceito de enhanced publication está relacionado ao aprimoramento das informações da pesquisa, com o uso de materiais extras, dados pós-publicação, banco de dados, entre outros, que apresentam ligação entre objetos. "Nesta definição, um objeto pode ser parte de um artigo, um conjunto de dados, uma imagem, um filme, um comentário, um módulo ou um link para informações em um banco de dados" (Woutersen-Windhouwer \& Brandsma, 2009, p. 31).

Bardi e Manghi (2014) estabelecem dois modelos de enhanced publications (Figura 1). Neste caso, observa-se que a parte narrativa está representada pelo artigo científico e a parte complementar é concebida a partir dos slides apresentados em uma conferência, do vídeo de apresentação e da planilha de dados relacionados, por exemplo. As duas publicações têm uma estrutura equivalente para o material suplementar, mas diferem na natureza da narrativa obrigatória: um único arquivo PDF versus um texto estruturado, ou seja, um hipertexto composto por várias subpartes interligadas, como: resumo, seções, figuras, tabelas, entre outros.

\footnotetext{
${ }^{1}$ Recuperado de https://plos.org/about/ (2020, novembro 13).

${ }^{2}$ E-prints são "recursos eletrônicos textuais que expressam um trabalho acadêmico original, destinado a ser lido por seres humanos, que apresenta algumas reivindicações acadêmicas e que geralmente contém uma interpretação ou uma análise de determinados dados primários" (Verhaar, 2007 como citado em Sales et al. (2013, p. 5).
} 


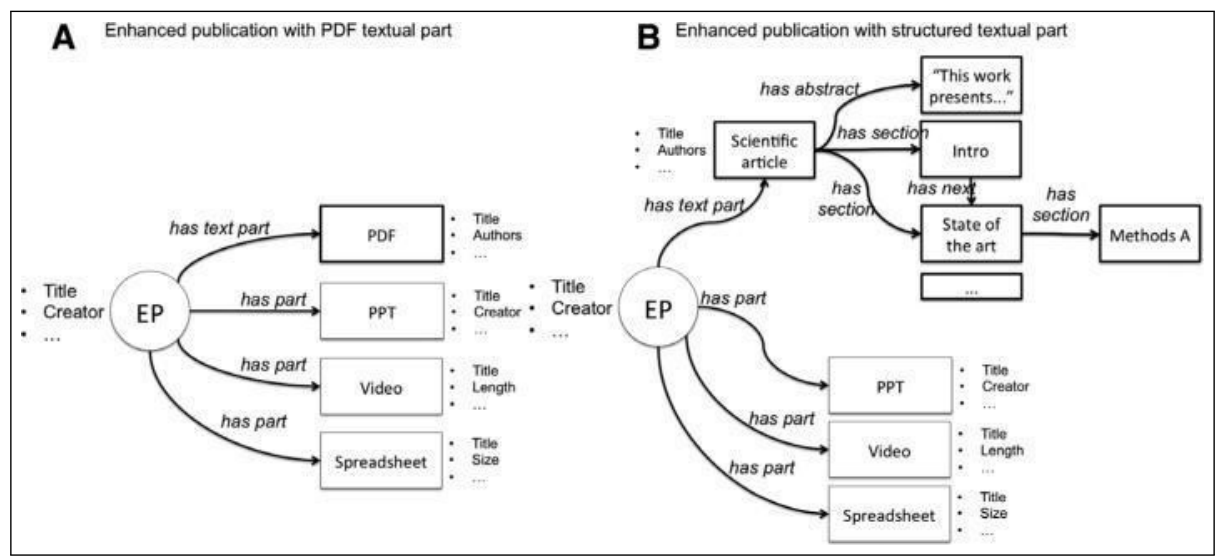

Figura 1. Exemplos de estruturas de enhanced publication.

Fonte: Bardi e Manghi (2014, p. 244).

Entende-se ainda, conforme Bardi e Manghi (2014), que o primeiro modelo, geralmente, é utilizado nas configurações de uma biblioteca digital, na qual o gerenciamento tradicional de artigos em PDF deve ser enriquecido com material suplementar. Já o segundo é mais apropriado para sistemas de informação que permitem uma experiência de leitura interligada dos artigos, por exemplo. Além disso, os autores apresentam uma classificação dos recursos do modelo de dados das enhanced publication (Figura 2).

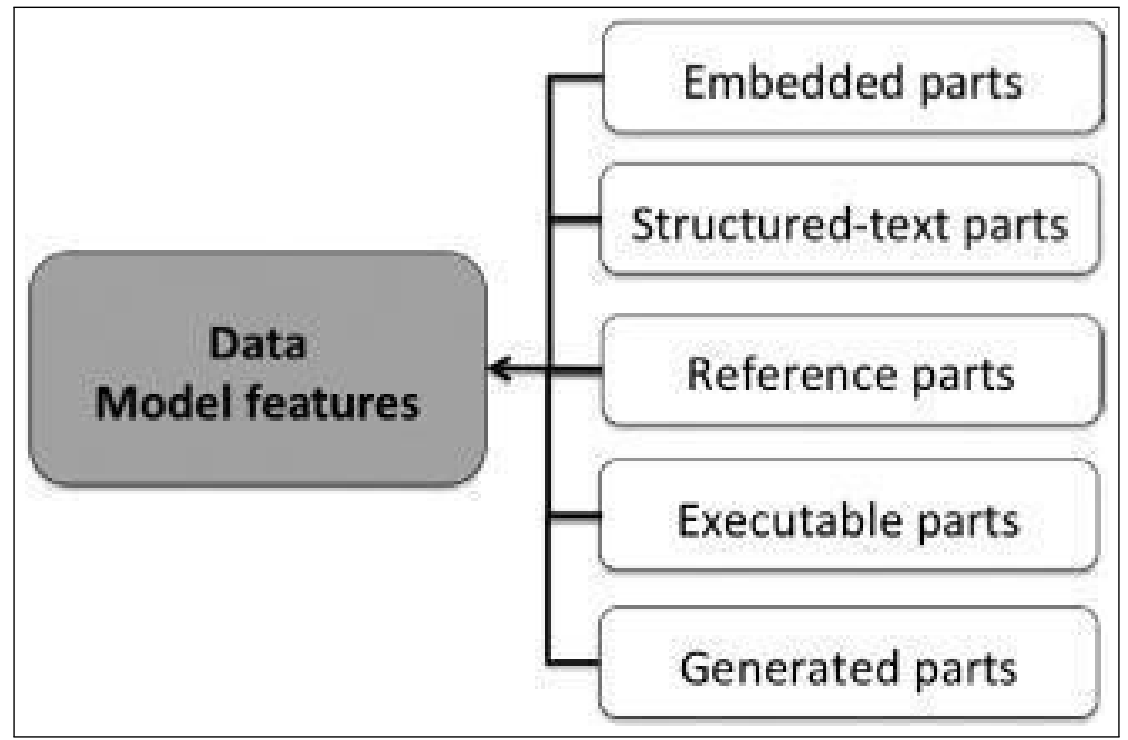

Figura 2. Recursos do modelo de dados das enhanced publication.

Fonte: Bardi e Manghi (2014, p. 245).

As partes integradas são aquelas que utilizam arquivos como materiais complementares. Por exemplo: slides de apresentação, apêndices para o artigo, descrição dos dados utilizados, imagens de alta resolução, tabelas que não podem ser inseridas totalmente na publicação digital devido aos limites de página, entre outros. Já as partes de texto estruturado constituem-se por meio de uma estrutura editorial que prevê subcomponentes textuais. Neste caso, os modelos de dados definem com precisão as relações entre as subpartes e podem incluir metadados (capítulo, seção, tabela) para dar suporte às funcionalidades avançadas de visualização e leitura (Bardi \& Manghi, 2014).

Considerando as limitações impostas às partes integradas, as partes de referência utilizam URLs para objetos externos, que apresentam links para resultados de pesquisas remotas, como conjunto de dados, citações, sites, apresentações e demais materiais adicionais ao conteúdo principal. Por sua vez, as partes executáveis fazem uso de softwares que possibilitem a reprodução do experimento, permitindo a comparação dos resultados encontrados com os apresentados no artigo, validando assim as decorrências da pesquisa. Por fim, as partes geradas contêm referências que se vinculam a bancos de dados externos, criando tabelas dinâmicas que são constantemente atualizadas (Bardi \& Manghi, 2014).

Salienta-se que o surgimento das publicações ampliadas foi impulsionado pela constatação de que a publicação tida como tradicional limitava-se à tentativa de incorporar os resultados de todo o processo de pesquisa científica (Verhaar, 2008 como citado em Sales, 2014). Nestas condições, a enhanced publication tem como missão agregar 
o maior número de recursos e/ou elementos que possam ampliar as conexões realizadas pelos pesquisadores, com o objetivo final de disseminar a Ciência de forma rápida e eficiente.

Desta forma, "uma publicação ampliada é uma nova forma de publicação onde a forma tradicional (um relatório, um artigo, um livro) é enriquecida com informações adicionais" (Mucheroni, Silva, \& Paletta, 2015, p. 3). Com isso, é imprescindível considerar que, para que a publicação aprimorada cumpra sua função, os materiais complementares devem estar armazenados em locais apropriados. Sales (2014) ressalta que os repositórios temáticos, nacionais e internacionais, devem utilizar identificadores persistentes para preservar e difundir com eficiência esse tipo de conteúdo.

Ainda, de acordo com Arraiza, Gonçalez, e Vidotti (2019, p. 16), uma publicação ampliada constitui-se "no contexto da publicação digital como uma forma de publicação dos dados de pesquisa junto com a publicação textual, agregando valor ao produto final e mostrando, de forma transparente, os processos que levaram à obtenção dos resultados apresentados". Diante disso, as autoras consideram que este tipo de publicação pode ser tomado como um objeto composto, com certas particularidades que desenham um marco para os trabalhos e os periódicos científicos.

Além disso, compreende-se que a grande contribuição da enhanced publication ao universo do conhecimento científico reside na melhoria da experiência de leitura por meio da web, sendo a publicação ampliada a responsável pela promoção de inúmeras relações entre essas publicações e o conjunto universal de dados de pesquisa. A maioria dos autores estudados veem com bons olhos os avanços trazidos pelas publicações aprimoradas, embora considerem que sua utilização ainda está aquém de seu potencial.

\section{METODOLOGIA}

O presente artigo apresenta resultados de uma pesquisa de natureza qualitativa, em nível exploratório que, por meio de um levantamento, culminou em uma análise de conteúdo dos dados encontrados. Em um primeiro momento, foi realizada uma pesquisa exploratória em revistas científicas melhor pontuadas de acordo com a base de dados JCR. ${ }^{3}$ Entretanto, após buscas nos sites das revistas e das editoras melhor classificadas, foi possível perceber que estas não apresentavam quantidade significativa de características que definem uma enhanced publication.

Para a escolha do objeto de estudo, portanto, utilizou-se como critério o modelo (Figura 2) proposto por Bardi e Manghi (2014, p. 245), no qual as enhanced publications são "publicações digitais que consistem em uma parte narrativa obrigatória (a descrição da pesquisa realizada) mais partes 'relacionadas', como conjuntos de dados, outras publicações, imagens, tabelas, fluxos de trabalho, dispositivos". De acordo com os autores, as enhanced publications são compostas por duas partes: a primeira, obrigatória, relacionada à narração textual; e a segunda, facultativa, caracterizada por um conjunto de subpartes interligadas por relações semânticas.

Além disso, considerou-se a argumentação de Woutersen-Windhouwer e Brandsma (2009), que afirmam que o exemplo mais conhecido de enhanced publication é o PLOS ONE, jornal on-line internacional, revisado por pares e de acesso aberto, que publica trabalhos com a adição de notas online, comentários e avaliações. Partindo desse ponto, o objeto de estudo desta pesquisa é a plataforma Public Library of Science (PLOS $)^{4}$ que, após o levantamento realizado, se apresentou como o conjunto de periódicos científicos que melhor ilustra as especificidades de uma publicação ampliada. Buscou-se verificar e apresentar a estrutura da $P L O S$ para demonstrar como as características e as funcionalidades das enhanced publications são utilizadas por esta plataforma, que envolve um jornal, seis revistas científicas e quatro tipos de conteúdo extra.

Com base no levantamento ${ }^{5}$ realizado no portal PLOS, foi possível perceber que as revistas científicas apresentam os mesmos recursos, sendo o site de cada uma delas desenvolvido a partir de uma estrutura padrão. Desta forma, são consideradas como estruturas padrão as revistas científicas: PLOS Biology, PLOS Medicine, PLOS Computational Biology, PLOS Genetics, PLOS Neglected Tropical Diseases e PLOS Pathogens. Por outro lado, são avaliadas as características individuais do jornal PLOS ONE e dos conteúdos extras: PLOS Collections, PLOS Currents, PLOS Blogs e PLOS Research News.

A partir da definição de quais características identificam uma publicação como uma enhanced publication e, considerando todo o universo de características da comunicação na web, a gama de produtos editoriais existentes na internet e as possibilidades que as TICs trouxeram para a comunicação científica, o Quadro 1 reúne as categorias de análise utilizadas no presente estudo.

\footnotetext{
${ }^{3} \mathrm{~A}$ base estatística InCites Journal Citation Reports (JCR), da editora Clarivate Analytics, é um recurso que permite avaliar e comparar publicações científicas utilizando dados de citações extraídos de revistas acadêmicas e técnicas e o impacto destas na comunidade científica. Recuperado de jcr.clarivate.com (2020, novembro 13).

${ }^{4}$ Recuperado de https://plos.org/ (2020, novembro 13).

${ }^{5}$ Salienta-se que a coleta inicial dos dados aconteceu em 2017, uma vez que esta pesquisa resulta de um Trabalho de Conclusão de Curso. Para este artigo, algumas informações foram atualizadas para melhor contextualizar os resultados.
} 


\begin{tabular}{|c|c|c|c|}
\hline AUTORES & CARACTERÍSTICA & TIPOS E RECURSOS & DESCRIÇÃO \\
\hline \multirow{4}{*}{$\begin{array}{l}\text { Palacios (1999-2004); } \\
\text { Bardoel e Deuze (2001); } \\
\text { Mielniczuk (2005); } \\
\text { Terra (2006); } \\
\text { Canavilhas (2014) }\end{array}$} & \multirow[t]{4}{*}{ Hipertextualidade } & Não-linearidade & $\begin{array}{l}\text { Fragmentação de conteúdos; } \\
\text { informação organizada em } \\
\text { formato de pirâmide } \\
\text { invertida. }\end{array}$ \\
\hline & & Link conjuntivo e disjuntivo & $\begin{array}{l}\text { Conjuntivo: mesma janela } \\
\text { do navegador com conteúdo } \\
\text { diferente. } \\
\text { Disjuntivo: quando se abre } \\
\text { uma janela menor ou outra } \\
\text { janela do navegador. }\end{array}$ \\
\hline & & Link interno e externo & $\begin{array}{l}\text { Interno: remete para lexias } \\
\text { dentro do próprio site. } \\
\text { Externo: direciona para } \\
\text { lexias externas ao site. }\end{array}$ \\
\hline & & $\begin{array}{l}\text { Link editorial, de serviços } \\
\text { e publicitário }\end{array}$ & $\begin{array}{l}\text { Editorial: pertence ao } \\
\text { conteúdo informativo do site. } \\
\text { De serviços: direciona para } \\
\text { serviços oferecidos pelo site. } \\
\text { Publicitário: remete à } \\
\text { publicidade de empresas } \\
\text { anunciantes. }\end{array}$ \\
\hline \multirow{7}{*}{$\begin{array}{l}\text { Bardoel e Deuze (2001); } \\
\text { Palacios (1999-2004); } \\
\text { Canavilhas (2014) }\end{array}$} & \multirow{7}{*}{ Multimidialidade } & Texto e fotografia & $\begin{array}{l}\text { Texto: elemento de } \\
\text { contextualização e de } \\
\text { documentação. } \\
\text { Fotografia: elemento de } \\
\text { ilustração. }\end{array}$ \\
\hline & & $\begin{array}{l}\text { Gráfico, iconografia e } \\
\text { ilustração estática }\end{array}$ & $\begin{array}{l}\text { Sinais que ilustram e orientam } \\
\text { o leitor em relação ao } \\
\text { conteúdo. }\end{array}$ \\
\hline & & Vídeo e animação digital & $\begin{array}{l}\text { Vídeo: imagens em } \\
\text { movimento. } \\
\text { Animação digital: imagens e } \\
\text { ilustrações geradas pelo } \\
\text { computador com efeitos } \\
\text { de movimento. }\end{array}$ \\
\hline & & Discurso oral & $\begin{array}{l}\text { Falas captadas, voz off e } \\
\text { legendas. }\end{array}$ \\
\hline & & Música e efeitos sonoros & $\begin{array}{l}\text { Peças multimídia que } \\
\text { utilizam áudio. }\end{array}$ \\
\hline & & $\begin{array}{l}\text { Folhas de estilo, } \\
\text { apresentações e descrições } \\
\text { textuais da pesquisa } \\
\text { (quadros, seções, tabelas) }\end{array}$ & $\begin{array}{l}\text { Elementos utilizados para } \\
\text { resumir ou ilustrar os } \\
\text { conteúdos. }\end{array}$ \\
\hline & & Vibração & $\begin{array}{l}\text { Sinais de alerta bastante } \\
\text { empregados em } \\
\text { dispositivos móveis. }\end{array}$ \\
\hline \multirow{2}{*}{$\begin{array}{l}\text { Palacios (2004-2014); } \\
\text { Bardoel e Deuze (2001); } \\
\text { Terra (2006); } \\
\text { Canavilhas (2014); } \\
\text { Valle (2016) }\end{array}$} & \multirow[t]{2}{*}{ Interatividade } & Seletiva & $\begin{array}{l}\text { Opções de acesso, como } \\
\text { menu e motores de busca. }\end{array}$ \\
\hline & & Comunicativa & $\begin{array}{l}\text { Interação } \\
\text { entre os sujeitos, } \\
\text { como comentários } \\
\text { e fóruns. }\end{array}$ \\
\hline
\end{tabular}




\begin{tabular}{|c|c|c|c|}
\hline \multirow{2}{*}{$\begin{array}{l}\text { Palacios (2004-2014); } \\
\text { Terra (2006); } \\
\text { Canavilhas (2014) }\end{array}$} & \multirow[t]{2}{*}{ Instantaneidade } & Tempo real & $\begin{array}{l}\text { Notícias de última hora, } \\
\text { atualizações imediatas } \\
\text { e notas curtas. }\end{array}$ \\
\hline & & Atualização contínua & $\begin{array}{l}\text { Disponibilização de novas } \\
\text { informações em tempo real. }\end{array}$ \\
\hline \multirow[t]{2}{*}{$\begin{array}{l}\text { Palacios (2004-2014); } \\
\text { Canavilhas (2014). }\end{array}$} & \multirow[t]{2}{*}{ Memória } & Banco de dados & $\begin{array}{l}\text { Conjunto de informações que } \\
\text { podem ser arquivadas, } \\
\text { indexadas e resgatadas por } \\
\text { determinados procedimentos } \\
\text { técnicos. }\end{array}$ \\
\hline & & Mecanismos de busca & $\begin{array}{l}\text { Sistemas que permitem a } \\
\text { localização de informações } \\
\text { nos bancos de dados. }\end{array}$ \\
\hline \multirow{7}{*}{$\begin{array}{l}\text { Palacios (2004-2014); } \\
\text { Bardoel e Deuze (2001); } \\
\text { Canavilhas (2014). }\end{array}$} & \multirow{7}{*}{ Personalização } & Customização do conteúdo & $\begin{array}{l}\text { Personalização de acordo com } \\
\text { os interesses individuais do } \\
\text { usuário. }\end{array}$ \\
\hline & & Resposta & $\begin{array}{l}\text { Capacidade de adaptação das } \\
\text { páginas da web para diferentes } \\
\text { dispositivos. }\end{array}$ \\
\hline & & $\begin{array}{l}\text { Alterar com base na } \\
\text { hora do dia }\end{array}$ & $\begin{array}{l}\text { O conteúdo se adapta de } \\
\text { acordo com o tempo e } \\
\text { as necessidades do usuário. }\end{array}$ \\
\hline & & Interação significativa & $\begin{array}{l}\text { Apresenta o conteúdo de } \\
\text { novas formas, de } \\
\text { acordo com os comentários } \\
\text { do usuário. }\end{array}$ \\
\hline & & Ajuda na decisão & $\begin{array}{l}\text { Oferece mecanismos que } \\
\text { auxiliam o usuário a } \\
\text { visualizar as consequências } \\
\text { de suas ações. }\end{array}$ \\
\hline & & Calibração e algoritmos & $\begin{array}{l}\text { Permite ao usuário visualizar } \\
\text { os dados recentes sempre que } \\
\text { estiverem disponíveis. }\end{array}$ \\
\hline & & Adaptável para mudar & $\begin{array}{l}\text { Pré-disposição para } \\
\text { modificações, imprescindíveis } \\
\text { à inovação dos sistemas de } \\
\text { personalização. }\end{array}$ \\
\hline \multirow[t]{2}{*}{$\begin{array}{l}\text { Terra (2006); } \\
\text { Canavilhas (2014) }\end{array}$} & \multirow[t]{2}{*}{ Ubiquidade } & Dispositivos móveis & $\begin{array}{l}\text { Permite o acesso às } \\
\text { informações de forma } \\
\text { interativa, em tempo real e } \\
\text { de qualquer lugar. }\end{array}$ \\
\hline & & Mídia gerada pelo consumidor & $\begin{array}{l}\text { Os usuários expressam } \\
\text { suas opiniões e replicam } \\
\text { em suas redes sociais. }\end{array}$ \\
\hline
\end{tabular}

Quadro 1. Categorias de análise das enhanced publications.

A seguir, apresentam-se os resultados e as discussões da análise desta pesquisa.

\section{RESULTADOS E DISCUSSÕES}

A partir da definição de quais características identificam uma publicação como uma enhanced publication e considerando o levantamento realizado na biblioteca digital de periódicos $P L O S$, são apresentados, na sequência, alguns recursos que podem ser utilizados por uma publicação científica ampliada, analisando, especialmente, como se dá a apropriação, por esta plataforma, das particularidades disponibilizadas pela web. No que diz respeito à característica de hipertextualidade, primeiramente é preciso destacar que a principal vantagem estabelecida para a comunicação científica está no fato de a navegação se apresentar não-linear (Terra, 2006). O usuário PLOS pode construir por si mesmo os caminhos de acesso aos conteúdos disponibilizados, o que permite ao usuário buscar informações de acordo com suas áreas de interesse de forma bastante interligada e dinâmica.

Quanto aos recursos de navegação, foram analisados os links apresentados como soluções que aperfeiçoam a experiência do usuário na procura de informações. Desta forma, na $P L O S$, os artigos das revistas científicas apresentam uma espécie de menu lateral, com a organização do conteúdo de tal forma que o leitor possa avançar 
ou retornar aos textos, sem necessariamente realizar uma leitura linear, o que se evidencia por meio dos links conjuntivos (Mielniczuk, 2005). Ao mesmo tempo, um menu superior permite que o usuário acesse uma série de dados que compreendem: o texto do artigo; as informações sobre o(s) autor(es); as métricas, tais como número de visualizações e de citações; os comentários; e os conteúdos relacionados que foram publicados em outras mídias (Figura 3).

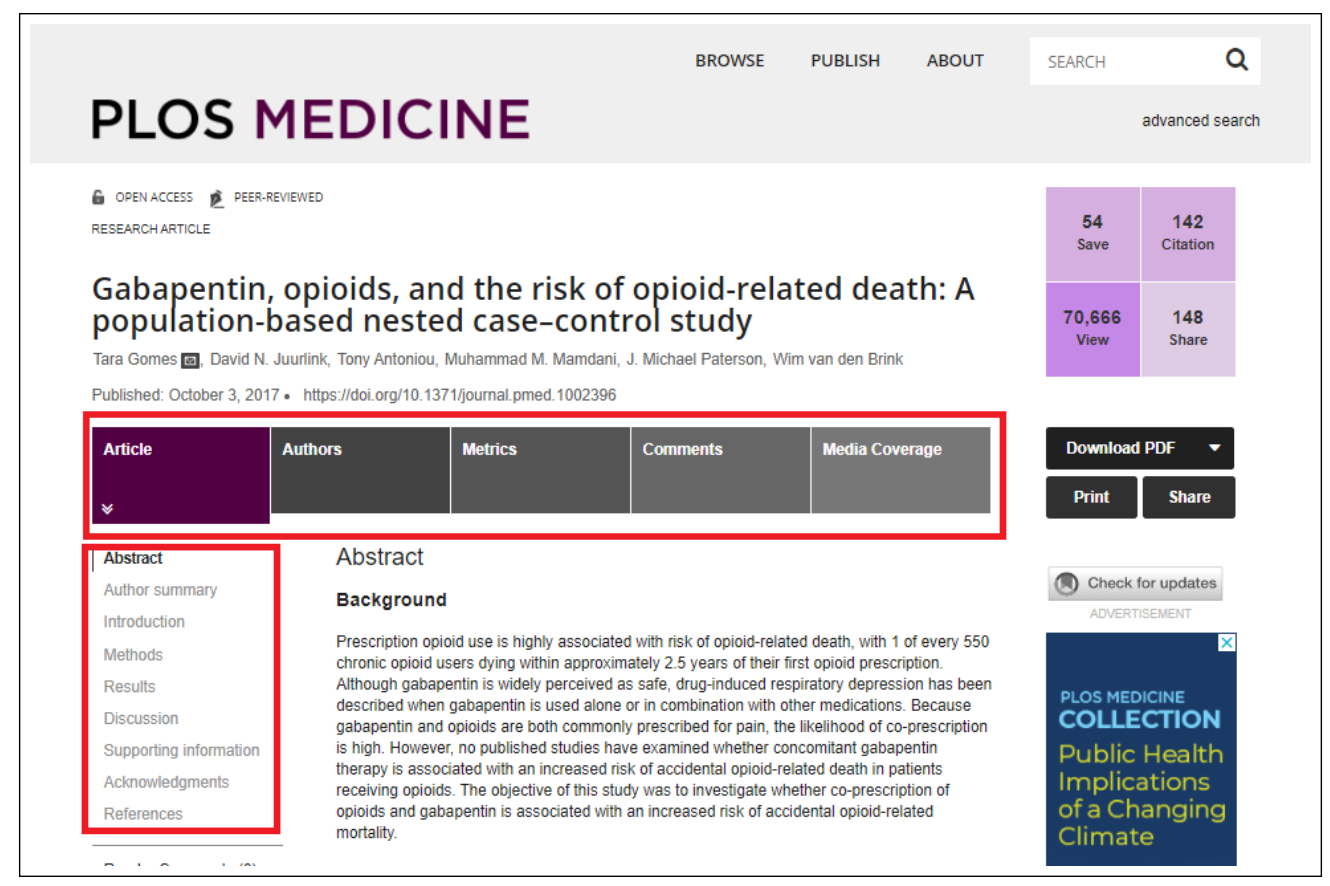

Figura 3. Exemplos de links conjuntivos (PLOS Medicine).

Fonte: http://journals.plos.org/plosmedicine/article?id=10.1371/journal.pmed.1002396 (2020, dezembro 10).

No que tange aos links disjuntivos (Mielniczuk, 2005), verificou-se que as figuras encontradas ao longo de um artigo, por exemplo, podem ser visualizadas através de miniaturas no próprio corpo do texto ou, se o leitor preferir clicar na imagem, expandidas em tela cheia. Neste caso, é possível acessar alguns comandos como: zoom, anterior/próxima, todas as imagens e download nos formatos PPT, PNG e TIFF. Outra facilidade encontrada é que, ao fechar esta janela, o leitor retorna ao texto, prosseguindo com sua leitura. Além disso, logo abaixo do título do artigo, onde estão citados o(s) nome(s) do(s) autor(es), é possível abrir um pop-up com endereço de e-mail, afiliações de trabalho e link para perfil na ORCID, por exemplo.

Em relação ao universo da abrangência (Mielniczuk, 2005), observam-se links internos nas páginas dedicadas aos autores, por exemplo, nas quais é possível verificar links que redirecionam o pesquisador para os critérios editoriais dos periódicos. Já os links externos são encontrados nos artigos das revistas científicas que apresentam links que redirecionam o pesquisador para conteúdo fora do portal PLOS. Neste caso, o leitor migra para o site do Creative Commons, e para os sites onde estão hospedados os trabalhos citados nas referências, como, por exemplo, o PubMed/NCBI e o Google Acadêmico. Considerando o tipo de informação, observa-se a presença de links editoriais na maioria dos textos das revistas $P L O S$, uma vez que este é o diferencial das publicações científicas.

No que se refere à característica multimidialidade (Bardoel e Deuze, 2001; Canavilhas, 2014; Palacios, 1999; 2004), no caso da PLOS, percebe-se que o texto compreende a maioria dos conteúdos veiculados no portal, sendo os artigos apresentados de forma tradicional na estrutura das informações textuais. Para complementar o texto, normalmente, usa-se uma fotografia, que tem o objetivo de ilustrar ou ainda de legitimar o contexto do tema que está sendo abordado. No caso da divulgação da Ciência, a fotografia torna-se um diferencial para a disseminação das pesquisas e dos processos que acontecem dentro dos laboratórios (Salaverría, 2014). Também é preciso destacar os gráficos, cujo uso, conforme Salaverría (2014), está cada vez mais comum devido a sua evidente capacidade de elucidar e resumir informações. No caso da divulgação da Ciência, o gráfico é um dos elementos-chave para o entendimento dos resultados da pesquisa, sendo, portanto, um dos recursos mais utilizados para demonstrar dados coletados e tabulados pelos pesquisadores em seus estudos, conforme ilustrado na Figura 4, que representa um gráfico retirado de um dos artigos da PLOS Genetics. 


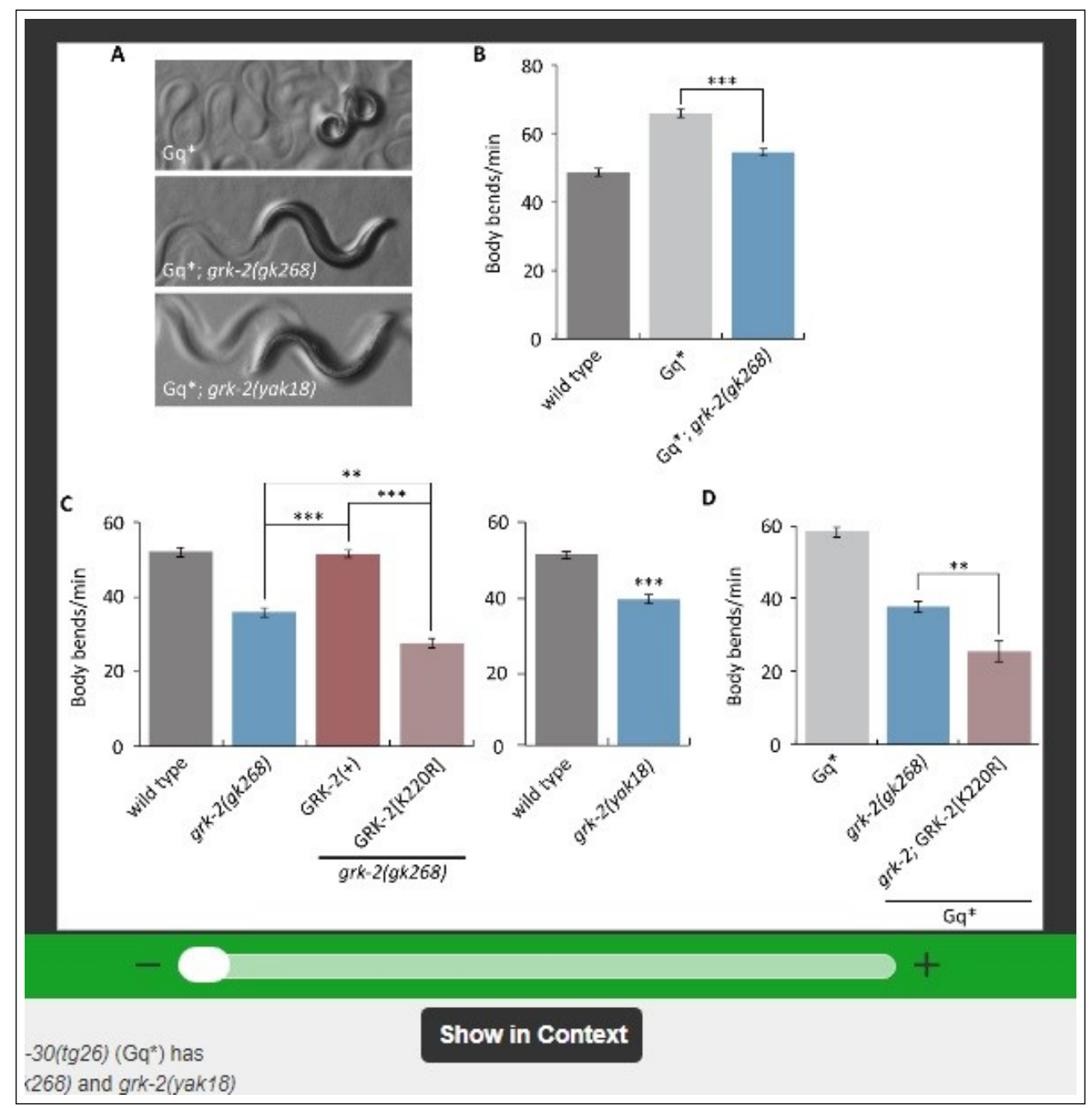

Figura 4. Exemplo de gráfico (PLOS Genetics).

Fonte: http://journals.plos.org/plosgenetics/article?id=10.1371/journal.pgen.1007032 (2020, dezembro 10).

Da mesma forma, os vídeos aparecem como um recurso de destaque na divulgação do conhecimento científico. No caso da PLOS, os vídeos são publicados originalmente em um canal no YouTube para, posteriormente, serem inseridos no corpo do texto no site. Entre as principais vantagens desta sistemática, está o fato de o próprio YouTube organizar os vídeos por ordem cronológica, ou de os editores poderem criar listas temáticas dentro do canal, o que facilita a recuperação das informações. Mais um recurso empregado pela $P L O S$ é a animação digital. Neste caso, pode-se citar como exemplo um artigo em que foi utilizada a técnica de imagem em 3D de alta resolução, que permitiu aos cientistas identificar duas novas espécies de formigas, cujas espinhas únicas inspiraram os pesquisadores a nomeá-las de dragões, baseados na série Game of Thrones. Desse modo, o indicador de visibilidade está na exploração de conteúdos com o uso de recursos que ampliam as possibilidades de visualização, como a mídia 3D. Fica clara, portanto, a potencialidade dos recursos de multimidialidade e de convergência, uma vez que os cientistas estão utilizando como referências produtos midiáticos que, em um primeiro momento, não apresentaram relação com o assunto estudado.

Cabe destaque também à utilização de discurso oral. Um dos blogs hospedados no PLOS Blogs é o PLOScast, que apresenta entrevistas em formato de podcasts com formadores de opinião, sobre o desenvolvimento editorial acadêmico, o futuro da academia e as experiências entre cientistas. Os arquivos em áudio estão organizados em episódios e foram publicados, originalmente, no SoundCloud, cujo link foi incorporado ao blog. No que concerne aos recursos sonoros, alguns efeitos são trabalhados nos próprios vídeos, como é o caso de um audiovisual, no qual um morcego é filmado rastreando um inseto em movimento e os sons ouvidos são reproduzidos por um detector de morcegos, o que possibilita que as vocalizações do animal sejam audíveis para os ouvidos humanos.

No tocante à interatividade (Rost, 2014), as revistas PLOS organizam as formas de contato em um menu lateral, no qual o usuário pode acessar, dentre outros: nome e e-mail do editor e escritórios PLOS. Desta forma, amplia-se a visibilidade da instituição, que promove o periódico e de seus próprios gestores. Além disso, em relação à interatividade seletiva, no final da página, existe um formulário de pesquisa, em que o leitor dá o seu feedback a respeito do motivo que o levou a acessar o site. Também é necessário considerar as diretrizes e as informações para autores como interatividade seletiva. A PLOS disponibiliza um sistema em que o pesquisador pode acessar três tipos de conteúdo: 1) envios: diretrizes para submissões; 2) política: pesquisas que envolvem 
seres humanos e animais, e direitos autorais, publicações éticas, entre outros; e 3) revisão e publicação do manuscrito: com esclarecimentos e orientações para o processo de revisão editorial e de pares.

Já no que se refere à interatividade comunicativa (Rost, 2014), destacam-se os ícones para as redes sociais e as formas de compartilhamento. A PLOS apresenta, na parte inferior de sua página inicial, três ícones que direcionam o usuário para suas páginas no Twitter, no Facebook e no LinkedIn. Individualmente, o jornal $P L O S$ $O N E$ e as demais seis revistas científicas PLOS têm em sua página inicial cinco ícones de "Fale Conosco". Estes dão acesso às seguintes informações: receber alertas por e-mail; documento RSS; Twitter (perfil individual de cada periódico); Facebook (PLOS); e aos PLOS Blogs.

Ainda, ao acessar um artigo específico, o leitor pode compartilhar o conteúdo, clicando no ícone à direita na página, com as seguintes redes: Reddit, Google +, StumbleUpon, Facebook, LinkedIn, CiteUlike, Mendeley, PubChase, Twitter e E-mail. É necessário destacar que o usuário pode fazer o download do arquivo em PDF (citação ou XML) e imprimir (também está disponível o recurso EzPrint). Vale ressaltar que, neste caso, o indicador de visibilidade está relacionado ao fato de a coletividade científica expandir suas pesquisas através do uso das mídias sociais, visando a aproximação com o leitor.

No que está relacionado à instantaneidade (Palacios, 2004), observando o princípio da atualização contínua, a PLOS mantém atualizados os números das métricas dos artigos. Ao acessar um artigo, o leitor poderá conferir, em ícones organizados à direita, o número de downloads, citações, visualizações e compartilhamentos deste texto. Estas informações são atualizadas automaticamente pelo sistema do site (Figura 5) e colaboram para a promoção da visibilidade da pesquisa e do próprio artigo.

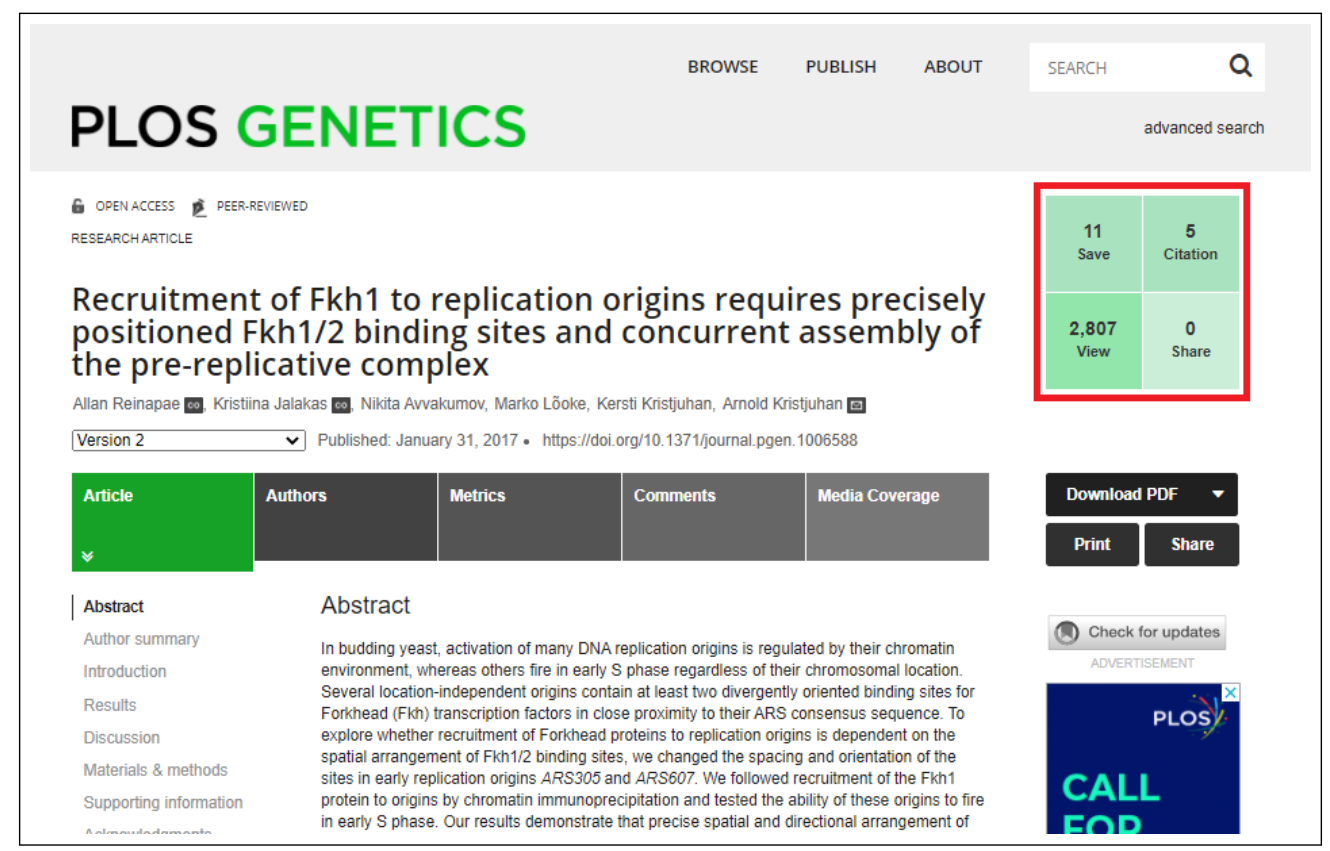

Figura 5. Exemplo de métricas de artigo (PLOS Genetics).

Fonte: http://journals.plos.org/plosgenetics/article?id=10.1371/journal.pgen.1006588 (2020, dezembro 10).

No que corresponde à memória (Palacios, 2004), é preciso destacar o funcionamento e a importância dos mecanismos de busca nos sites de comunicação científica. A PLOS é considerada um portal, uma vez que congrega várias páginas de conteúdo e serviços distintos e, ao mesmo tempo, afins. Em cada página, é possível perceber que os editores trabalham os mecanismos de busca com o intuito de melhor adaptar o conteúdo ao alcance do usuário, possibilitando a recuperação e a visibilidade das pesquisas.

Desta forma, em relação ao jornal PLOS ONE, é possível perceber que, por meio do menu Browse, o leitor pode fazer sua busca no acervo, que conta com 243.312 artigos (em 10 de dezembro de 2020). Para isso, há um filtro dividido em onze áreas e, ao selecionar uma área, o usuário ainda pode seguir sua busca por meio de uma nova lista com subáreas, sendo que estas variam em número dependendo da qual estão vinculadas. No caso das revistas científicas da PLOS, os mecanismos de busca para consulta ao acervo levam em conta os anos de publicação, podendo o leitor acessar os exemplares mensais através de uma linha do tempo de acordo com o exemplo da Figura 6. 


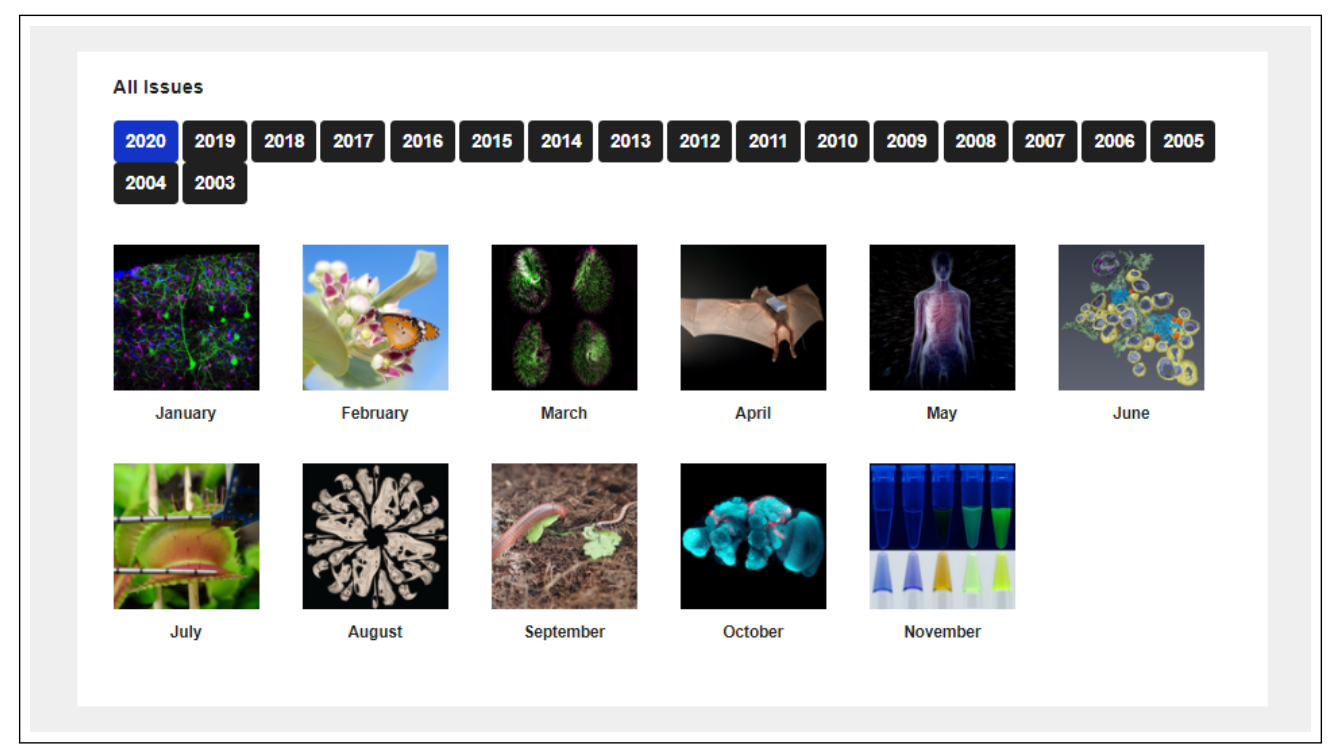

Figura 6. Consulta ao acervo da revista científica PLOS Biology.

Fonte: http://journals.plos.org/plosbiology/volume (2020, dezembro 10).

Ainda em relação à memória, é preciso perceber que, ao disponibilizar seu acervo organizado por ano, por exemplo, a PLOS está preservando a memória da pesquisa científica nas áreas em que se dedica, através da formação de um grande banco de dados online. Da mesma forma, este recurso possibilita a fácil recuperação de informações e, ao mesmo tempo, o compartilhamento de dados.

No que tange à personalização (Bardoel e Deuze, 2001; Canavilhas, 2014; Palacios, 1999; 2004), não foram encontrados elementos estéticos que possam ser customizados na estrutura geral dos sites. Talvez este recurso possa estar disponível aos usuários que têm login e senha cadastrados. Entretanto, se for considerado o acesso aos conteúdos disponíveis, o cadastramento para o recebimento de informações por e-mail, por exemplo, pode ser considerada uma forma de personalização. Da mesma maneira, os mecanismos de busca permitem que o usuário trace seu próprio percurso de leitura de acordo com seus interesses, personalizando sua navegação ao longo das páginas.

Finalmente, no que diz respeito à ubiquidade (Canavilhas, 2014; Terra, 2006), identificou-se que os sites da $P L O S$ e de suas revistas científicas e conteúdos extras também foram pensados para se adequar à interface dos dispositivos móveis. A grande vantagem destes ajustes reside na melhor visualização dos conteúdos e dos sistemas de menus, facilitando o acesso às informações por parte do usuário. Assim, a visibilidade das pesquisas é facilitada pelo uso e adequação do layout aos dispositivos móveis.

A partir desta análise, percebe-se que a grande contribuição do desenvolvimento da web à comunicação científica reside na oferta de recursos para a divulgação dos resultados e avanços das pesquisas, tornando os periódicos científicos veículos com grande potencial para a ampliação da visibilidade da Ciência. Conclui-se o presente diagnóstico verificando que as características de uma enhanced publication podem criar muitas oportunidades para o tratamento e a divulgação da comunicação científica. A palavra de ordem, neste caso, é adequação, uma vez que o pesquisador/editor necessita estar atualizado a respeito dos recursos que são oferecidos pela internet, atento às potencialidades criadas pelas suas análises e cercado de profissionais capacitados, que possam dar suporte às práticas que serão adotadas pelo periódico na divulgação de suas pesquisas.

\section{CONSIDERAÇÕES}

Os resultados da pesquisa expostos neste artigo permitiram identificar e apresentar quais recursos da enhanced publication são utilizados pela plataforma $P L O S$, com o intuito de ampliar a visibilidade das publicações científicas. Da mesma forma, foi possível verificar como os periódicos científicos podem se utilizar destas particularidades das enhanced publications a partir do exemplo da PLOS e das suas múltiplas páginas de conteúdo sobre Ciência. Ainda que de forma modesta e isolada no cenário da comunicação científica na web, a PLOS torna-se um modelo a ser seguido, pois apresenta vários recursos das publicações ampliadas, de maneira bastante didática. Entende-se, entretanto, que outras possibilidades poderiam ser aplicadas, para potencializar os resultados de visibilidade.

Além disso, os resultados desta análise propõem uma espécie de caminho para que outros editores de comunicação científica possam entender o processo de criação de uma publicação ampliada e desenvolver seus próprios modelos a partir dos tipos e recursos aqui apresentados. Desta forma, a grande contribuição deste trabalho é apresentar para o pesquisador/editor as possibilidades de uso dos recursos competentes às enhanced publications. 
Averiguou-se também que hipertextualidade e multimidialidade caminham juntas para produzir uma publicação ampliada de qualidade, pois o texto agregado aos elementos multimídias agregam valor aos periódicos científicos aprimorados. A participação do leitor, através da interatividade proporcionada pela web, torna-se um diferencial para a comunicação científica, especialmente, pelo fato de potencializar a visibilidade das publicações a partir do compartilhamento de informações entre pares.

Destaca-se que se faz necessária a implementação dos recursos das enhanced publications para a ascensão da comunicação científica, visto que essas características ampliam as publicações científicas e potencializam a visibilidade dos envolvidos no processo de criação e produção de um periódico, sendo que todos devem estar engajados nesta construção. Da mesma maneira, trabalhar com estes recursos melhora a credibilidade das publicações, colaborando para a legitimação das revistas científicas. Por isso, espera-se que esta pesquisa possa abrir discussões a respeito dos princípios e uso das publicações ampliadas nas revistas brasileiras. 


\section{REFERÊNCIAS}

Ambinder, D. M. (2012). Artigos cientificos digitais na web: novas experiências para apresentação, acesso e leitura (Dissertação de mestrado, Departamento de Ciência da Informação, Universidade Federal Fluminense Niterói, Niterói, RJ, Brasil). Recuperado de https://app.uff.br/riuff/handle/ $1 / 352$

Arraiza, P. M., Gonçalez, P. R. V. A., \& Vidotti, S. A. B. G. (2019). Recomendações para a integração de publicações ampliadas em repositórios digitais confiáveis. Encontros Bibli: revista eletrônica de biblioteconomia e ciência da informação, 24 (55), 1-23. doi: https://doi.org/10.5007/15182924.2019.e58556

Bardi, A., \& Manghi, P. (2014). Publicações aprimoradas: modelos de dados e sistemas de informação. LIBER Quarterly, 23(4), 240-273. doi: http://doi.org/10.18352/lq.8445

Bardoel, J., \& Deuze, M. (2001). Network journalism: Converging competences of media professionals and professionalism. Australian Journalism Review, 23(2), 91-103. doi: http://doi.org/10.18352/lq.8445

Canavilhas, J. (2014). Hipertextualidade: novas arquiteturas noticiosas. In Webjornalismo: 7 caraterísticas que marcam a diferença (p. 3-24). Covilhã: Livros Labcom.

Degkwitz, A. (2016). Enhanced publications resp. future publications. In Proceedings of the unica scholarly communication seminar. Suíça, Lausanne. Recuperado de https://goo.gl/VMQKYy

Gomes, C. M. (2014). Comunicação científica: Cartografia e desdobramentos (Tese de Doutorado, Escola de Comunicação e Artes, Universidade de São Paulo, São Paulo, Brasil). Recuperado de https://bdpi.usp.br/item/002321102

Mielniczuk, L. (2005). O link como recurso da narrativa jornalística hipertextual. In Anais do congresso brasileiro de ciências da comunicação. Rio de Janeiro, RJ, Brasil. Recuperado de http://www.portcom.intercom.org.br/pdfs/ 160318299140382081603311405193211973269.pdf

Mucheroni, M. L., Silva, F. J. M., \& Paletta, C. F. (2015). Entre a publicação ampliada e a multimodalidade. In Anais do encontro nacional de pesquisa em ciência da informação. João Pessoa, PB, Brasil. Recuperado de http://repositorios.questoesemrede.uff.br/repositorios/ handle/123456789/2968

Palacios, M. (1999). O que há de (realmente) novo no jornalismo online? conferência proferida por ocasião do concurso público para professor titular na facom/ufba. Salvador, Bahia.

Palacios, M. (2004). Jornalismo online, informação e memória: apontamentos para debate. Revista PJ:BR, 4. Recuperado de https://goo.gl/qstWc8

Palacios, M. (2014). Memória: jornalismo, memória e história na era digital. In Webjornalismo: 7 caraterísticas que marcam a diferença (p. 89-110). Covilhã: Livros Labcom.

Rost, A. (2014). Interatividade: definições, estudos e tendências. In Webjornalismo: 7 caraterísticas que marcam a diferença (p. 55-88). Covilhã: Livros Labcom.

Salaverría, R. (2014). Multimedialidade: informar para cinco sentidos. In Webjornalismo: 7 caraterísticas que marcam a diferença (p. 25-51). Covilhã: Livros Labcom.

Sales, L. F. (2014). Integração semântica de publicações científicas e dados de pesquisa: proposta de modelo de publicação ampliada para a área de ciências nucleares (Tese de Douto- rado, Instituto Brasileiro de Informação em Ciência e Tecnologia, Universidade Federal do Rio de Janeiro, Rio de Janeiro, RJ, Brasil). Recuperado de https://ridi.ibict.br/bitstream/ 123456789/874/1/LUANA\%20SALES\%20D.pdf

Sales, L. F., Sayão, L. F., \& Souza, R. F. (2013). Publicações ampliadas: um novo modelo de publicação acadêmica para o ambiente de e-science. In Anais do encontro nacional de pesquisa em ciência da informação. Florianópolis, SC, Brasil. Recuperado de https://ridi.ibict.br/handle/123456789/454

Spyer, J. (2007). Conectado: o que a internet fez com você e o que você pode fazer com ela. Rio de Janeiro: Jorge Zahar.

Terra, C. F. (2006). Comunicação corporativa digital: o futuro das relações públicas na rede (Dissertação de mestrado, Escola de Comunicação e Artes, Universidade de São Paulo, São Paulo, Brasil). Recuperado de https://goo.gl/U3fkZZ

Valerio, P. M. (2006). Periódicos científicos eletrônicos brasileiros: ampliando visibilidade e acesso. In Anais do encontro nacional de pesquisa em ciência da informação. Marília, SP, Brasil. Recuperado de https://goo.gl/kdn26B

Valle, T. O. F. D. (2016). Cocriação de valor como estratágia de comunicação na sociedade em rede: estudo do programa cocriando natura (Dissertação de mestrado, Centro de Ciências Sociais, Universidade Federal de Santa Maria, Santa Maria, RS, Brasil). Recuperado de https://repositorio.ufsm.br/ handle/1/6373

Woutersen-Windhouwer, S., \& Brandsma, R. (2009). Enhanced publications, state of the art. In Enhanced publications: linking publications and research data in digital repositories. Amsterdam: Amsterdam University Press.

Woutersen-Windhouwer, S., Brandsma, R., Hogenaar, A., \& Hoogerwerf, M. (2009). Enhanced publications: linking publications and research data in digital repositories. Amsterdam: Amsterdam University Press.

Como citar este artigo (APA):

Avila, E. \& Bomfá, C. R. Z. (2021). Enhanced publications: um estudo da plataforma Public Library of Science (PLOS). AtoZ: novas práticas em informação e conhecimento, 10(1), 60 - 71. Recuperado de: http://dx.doi.org/10.5380/atoz.v10i1.76846 